\title{
Assessment of the NASA Space Shuttle Program's problem reporting and corrective action system
}

\author{
D. J. Korsmeyer ${ }^{\star}$, J. A. Schreiner \\ NASA Ames Research Center, MS 269-1 \\ Moffett Field, Ca 94035-1000 USA
}

\begin{abstract}
This paper documents the general findings and recommendations of the Design for Safety Program s Srudy of the Space Shuttle Program s (SSP) Problem Reporting and Corrective Action (PRACA) System. The goals of this Study were; to evaluate and quantify the technical aspects of the SSP S PRACA systems, and to recommend enhancements addressing specific deficiencies in preparation for future system upgrades. The Study determined that the extant SSP PRACA systems accomplished a project level support capability through the use of a large pool of domain experts and a variery of distributed formal and informal database systems. This operational model is vulnerable to staff tumover and loss of the vast corporate knowledge that is not currently being captured by the PRACA system. A need for a Program-level PRACA system providing improved insight, unification, knowledge capture, and collaborative tools was defined is this study.
\end{abstract}

Keywords: PRACA, NASA Space Shuttle, Problem Reporting, Databases

\section{INTRODUCTION}

The Space Shuttle Program s (SSP s) Problem Reporting and Corrective Action (PRACA) systems and their supporting infrastructure (used to report discrepancies, non-conformances, problems, track engineering dispositions, corrective actions and provide data for trend analysis and reporting) are an essential tool for managing Shuttle safety and readiness for flight. . The charter document that describes the requirements for the SSP PRACA system is NSTS 08126, Revision G [ref. 2]. Because of their importance, the PRACA systems have been the subject of several recent reviews aimed at improving the systems utility and improving the motivating requirements. In September 1999. NASA chartered the SIAT to provide an independent review of the Space Shuttle sub-systems and maintenance practices. The SIAT published its report in March 2000 [ref. 3]. In the SIAT report, several findings and recommendations were raised specifically regarding the SSP problem reporting practices and systems that may adversely affect Shuttle safety.

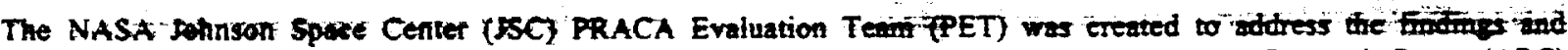
recommendations from the Shuttle Independent Assessment Team (SIAT), the initial NASA Ames Research Center (ARC) assessment comments, and other SSP sponsored PRACA audits and reviews. The PET was established by SSP Review Control Board Action S06034lR5(3-1) [ref. 1]. The PRACA Enhancement Pilot Study (the subject of this paper) was coordinated with the JSC PET, and as part of a new NASA Initiative - the Design for SafetyProgram (DFS). A team at NASA Ames Research Center performed the PRACA Enhancement Pilot Study.

This paper documents and provides a general overview of the technical evaluation of the existing operational SSP PRACA systems. The evaluation then generalizes the technical findings and recommends enhancements to improve this critical NASA distributed information system. The full results of the Study and the detail assessments of the above four areas are documented in the NASA Ames Research Center Technical Memorandum published on the Study [ref. 4]:

- User Interface

- Database and Data Management

- Network and System Architecture

- Problem Reporting Work Processes

A key element of the continuing success of the Space Shuttle Program and the operation of the multiple PRACA systems has been the dedicated and enthusiastic staff of NASA and its contractor team. The progress of this Study was greatly aided by the tremendously dedicated and hard working individuals supporting the Space Shuttle Program. Everyone we spoke to

' Correspondence: D. J. Korsmeyer, email: dkorsmever@arc nasa.gov 
through the course of this Study was highly cooperative and willing to assist us in completing this assessment and to ensure a continued safe Space Shuttle System.

The broad general assessment of the current PRACA systems is that they achieve sufficient project support capability through engagement of a large number of highly dedicated and competent staff. The systems are frequently redundant and not interconnected which produces inefficiencies and the potential for data loss and input error. The current approach employed in the PRACA deployment does not scale or adapt easily to changes in workforce or technology. Further, the exper knowledge that is required to utilize the PRACA systems is not captured or documented. As a result, the current PRACA system is not capable of supporting risk-assessment functions performed at the Program-level.

We believe that an Agency-wide NASA/Industry team in conjunction with the SSP PRACA workforce can bring together the required expertise, knowledge. and advanced IT capabilities necessary to achieve NASA s Information Management vision for PR.ACA. In so doing, PRACA will remain a critical and vital system, enabling a reduction in the risk and improvements in safety while supporting the Space Shuttle Program into the next decades.

\section{MOTIVATION AND APPROACH}

\section{The Creation of the SSP PRACA System}

In 1987, after the Challenger accident and in response to the Rogers Commission recommendation to provide NASA Space Shuttle management and decision makers with readily available, timely, and accurate data, the Program Compliance Assurance and Status system (PCASS) was formed. The PCASS is defined in document System Integrity Assurance PTogram. NSTS 07700 Volume XI, [ref. 6]

The NSTS 07700 goals were to impact Shuttle processing, safety, and readiness for flight by enabling continuous process improvements. The premise was that an overall PRACA System would allow users access to the current and historical data necessary to perform trend analysis and reporting to aid in the process planning and improvement. Currently, to provide the data necessary for this Program-wide view into the PRACA data, a combination of paper records, on-line databases from separate PRACA systems, and corporate/expert knowledge and skilled personnel are required.

PCASS is being re-hosted from the mainframe-based PCASS onto a Unix server with a browser interface. However, the NSTS 07700 goals of an interactive Shuttle data store for use in trending, safety and reliability analyses are not yet being realized.

\section{Assessment Approach}

The objective of the Study was to document a quantitative assessment of the technical and operational status of the SSP PRACA systems and elements. In addition to the as-implemented aspects, the team desired to understand the as used issues and challenges with the PRACA systems so that any recommendations, while technically feasible, can also be evaluated for their practicality and work environment utility.

The approach taken by the Study team was to interview, understand, and assess. This approach required multiple site visits, telecons, and interviews with as many of the people involved in the PRACA system as possible (managers, users, customers, etc). The team consistently noted the support and cooperation by the NASA and contractor staff throughout the SSP PRACA system. This was fairly unique in the team s experience to see such cross-center cooperation and enthusiasm for progress towards a common goal. All of the team $s$ requests for information, documentation and time were professionally addressed and met the team s needs.

The quantitative technical assessment began in March 2000. The Team visited and interviewed PRACA systems owners and users at JSC. KSC, and MSFC. The purpose of these meetings was to understand how the SSP elements collect, manage, and use the problem reporting data. The team also interviewed multiple safery, reliability, quality and mission assurance users of the PRACA data to determine the desires and implicit requirements for the PRACA systems. As part of the interview process, the team collected available system documentation recommended by the contacts. 


\section{STATE OF THE SSP PRACA SYSTEM}

This section details the state of the SSP PRACA systems. This information was collected from the interviews with various PRACA system owners and the ProjectElement level and analysis of available PRACA documentation.

\section{PRACA System Overview}

The NSTS 08126 Revision G document was signed on February 2. 1996. That document provides the minimum requirements and responsibilities applicable to all SSP PRACA systems, as required by NHB 5300.4(1D-2). Safety, Reliability. Maintainability and Quality Provisions for the SSP [ref. 7]. The objectives of NSTS 08126 Revision G are to:

a. Establish uniform standards to ensure safety, reliability, and quality of SSP.

b. Establish the requirements/procedures to assure problems are dispositioned prior to flight.

c. Ensure appropriate corrective action is taken in a timely and cost effective system.

d. Provide the problem data necessary to support engineering analyses and logistics management.

The NSTS 08126 Revision G does not address the issues or requirements for extracting the data necessary for trend analysis and reporting. While it does provide the data for engineering analyses and logistics, this has not proved sufficient to the safety analysts and has necessitated having adjunct data sources and databases to perform risk and reliability trend analyses.

NSTS 08126 Revision $G$ has established some uniform standards for ensuring safery, reliability, and quality of SSP, but has not required or documented the critical information and data standards. These include data management standards, such as field naming and database schema, that would enable the development of common SSP-wide PRACA data systems. Such a system is discussed in the recommendations, and is what the current implementation of the SSP PRACA lacks. The use and design of the existing PRACA systems. described in the following sections. points to the innate desire of the SSP for a unified access to PRACA data.

Implementation of SSP PRACA

The current SSP PRACA System is a collection of computer hardware, software, networks, and databases, as well as extensive paper files distributed across several NASA, USA and other contract support sites. These separate PRACA systems are managed by various teams of individuals whose job is to maintain the systems. keep the databases current. and assist in the extraction of data for trending and reporting. One of the key components contributing to the success of the current PRACA systems is this support staff that forms the extensive corporate and institutional knowledge necessary to analyze. reduce, produce conclusions, and report results from the PRACA databases. Without these highly trained and expett starf, the utility of the PRACA data is reduced significantly.

The data management capability behind the SSP PRACA System is a conglomeration of Project-level database systems. Many of the component systems are designed as workflow management tools with strict requirements for streamlining accurate and timely problem resolution. Other systems are focused upon capturing the problem report data at a high fidelity useful in safety analysis and data mining applications. It is these two dissimilar requirements (workflow efficiency vs. extensive problem documentation) that are at odds in the current SSP PRACA systems.

For the purposes of SSP use, the PRACA system is viewed as a collection of domain-expert managed systems. At the time of this Study most of the data from all but one of the Project PRACA systems could be queried through a centralized data warehouse for summary and condensed report viewing. This warehouse is called the Advanced Data Acquisition and Management (ADAM) [ref. 7]. However, as noted by the SIAT, ADAM cannot be data mined or navigated by the non-expert user. As a result, the SSP uses the PRACA systems principally by contacting the appropriate responsible domain experts and requesting that a report or data trending exercise be produced for the Program office. Then, a team of domain experts at JSC. KSC, MSFC and other support sites accesses the PRACA component databases, other experts, additional off-line databases, and paper records to produce a report for SSP. These reports are usually placed in the PRACA systems and become part of the on-line record. It is important to note that reports of any sophistication (i.e., data mining, detailed cross PRACA correlations etc.) cannot be generated in real time using the on-line PRACA systems alone. 


\section{PRACA SYSTEM GENERAL FINDINGS}

Upon completion of the on-site interviews, reviews and technical assessments of the multiple PRACA systems. the team identified a set of general findings, and a set of findings specific to the four technology areas identified in our Approach. The overall assessment of the existing PRACA systems is that they are inefficient and potentially vulnerable to data loss and input error. The current approaches do not scale or adapt easily to changes. The expert knowledge that is required to utilize the PRACA systems is not captured or documented. Overall, the existing PRACA systems are incapable of supporting Programlevel risk assessment. These general and specific technical findings are discussed in the following sub-sections.

Current PRACA Does Not Meet SSP Needs

The PRACA systems in use by the SSP are not sufficient to meet the SSP current and future needs (as expressed by NASA SSP management, the SSP requirements documentation (i.e., NSTS 07700 Volume XI), and from a sustainable workforce perspective). This general finding is primarily due to lack of clarity in expression of a vision for PRACA, which is the result of three primary causes.

1. The SSP is currently able to accomplish its PRACA Shuttle management tasks, yielding the perception that PRACA works. Because of this perception, questions addressing the essential functional, systematic and architectural aspects of PRACA are not being asked.

2. The SSP requirements documentation does not preclude the use of data outside the formal PRACA data systems. This enables a capability much greater than would be possible if the users were restricted to PRACA system data only. As a result, the SSP has not been confronted with the data limitations of the current PRACA systems.

3. The current SSP PRACA system is perceived as having a system-wide, user-navigable data warehouse capability. This perception is further reinforced by the use of domain experts who extract data and reports from the various Project-Element-designed and supported systems. This gives the impression of a system-wide data mining and navigation capability. Because of this perception, the SSP is not articulating and advocating a data warehouse rearchitecture to PRACA (believing it already has one).

These three reasons are described further in the following sub-sections:

The Perception That PRACA Works.

The premise of the PRACA capabilities described in NSTS 07700 Volume XI sections 3.4 and 4.1.5.x is to integrate program element trend systems, perform analysis, and provide data formatted for management visibility to support Shurtle Safety Assessments. The SSP does this by reliance upon subsystem domain experts, and the performing safety organizations skilled personnel, to report starus and trends to the Program office. The domain experts possess substantial intstitutional knowledge and are able to draw from information outside the PRACA data systems. The reports provided to the SSP therefore are based on large amounts of data outside of the PRACA systems. The loss of the domain experts and the external data sources would significantly degrade the quality of the PRACA reporting and trending. The use of domain experts enhances the quality of the knowledge extracted from the PRACA systems, giving the Program Office the mistaken impression that the PRACA systems alone possess equivalent data and knowledge-generation capabilities as those presented in the reports.

For the trending and analysis groups to perform their tasks using current PRACA, it is sometimes necessary to filter or correct the data and generally augment PRACA data to produce meaningful results. Several NASA safety, reliability and quality assurance (SR\&QA) groups identified this practice as necessary to perform meaningful trending and analysis for the Program office. The JSC Shuttle Orbiter SR\&QA group. for example, created and manages the Shuttle Risk and Reliability Analysis Database (SRRAD) and created manual and automated filtering and data processing routines for this purpose. The SRRAD database and data filtering and correction process are examples of data and expertise upon which the SSP reports are dependent but are not part of any of the formal PRACA systems.

\section{The Use of Non-PRACA Data in Reports}

The use of data outside the formal PRACA system has allowed the evolution of informal data systems upon which the SSP depends. These informal databases exist, in many cases, at the expense of a proper upgrade of the formal PRACA systems. 
The base SSP PRACA requirements documentation (NSTS 07700) imply that a PRACA System will be the official repository for all data necessary to manage Shuttle problem reporting and corrective action assessment, diagnosis, and trending. However, the NSTS 08126 Revision G requirements also turn responsibility for generating the status, assessment and diagnosis over to Project Element domain experts with no constraints on the data they are permitted to use to generate the reports. In order to produce the best reports possible, the experts naturally draw from all the information they can access.

The dependence of the SSP on domain experts to produce reports is codified in the requirements document (i.e., it is the responsibility of the domain expert centers to manage their subsystems and report their data to the program office). This has effectively hidden the lack of stand-alone capability the SSP desires for PRACA. It has also hidden the fact that the PRACA system cannot be data mined by the non-expert users. The expert users can manually navigate the various information systems using implicit and innate familiarity with the data, thus giving the appearance of a data mining capability. A new or inexperienced user would not have the knowledge or context to understand the syntax, or codes used for each of the PRACA systems. Indeed, an expert on one SSP PRACA system is not an expert on all PRACA systems.

As with any large requirements-based information system, the domain experts have found that it is easier to build and manage an informal database to augment PRACA data outside of the SSP PRACA purview than it is to upgrade the SSP PRACA databases. For example, as mentioned in the section above, the JSC SR\&QA group performs trending and analysis for the Program office by creating and managing the SRRAD database. The SSRAD database is used in conjunction with the PRACA data but is not part of any formally recognized PRACA system. These adjunct PRACA data sources and their requirements need to be addressed in the overall SSP PRACA picture

\section{The Perception that PRACA is a Navigable Warehouse of Data}

The SSP has expressed wishes to incorporate the individual PRACA systems into a unified program data warehouse but has not initiated a major re-architecture or training program to elevate the focus from the SSP Element/Project level to the Program level. The Project-level or component PRACA systems resident at JSC, KSC, and MSFC have been designed and implemented by the PRACA domain experts at each center. These systems were developed to meet their individual task and project support needs as stand-alone systems. The inevitable local prioritization of tasks results in no two systems having the same global motivation for existence or the same technical implementation basis. The Project-level systems are not innately amenable to hybrid data warehouse architecture.

USA s ADAM is a good first attempt to provide unified PRACA data and some associated information. The ADAMl effort sidesteps the individual Project PRACA system differences by duplicating the data onto one site, where an interface and mapping can insulate the user from systern-to-system navigation difficulties. ADAM cannot, however, resolve the issues of data quality, consistency, integrity, and breadth, which are limited by source PRACA systems. ADAM is increasing tata visibility and bringing attention to data errors and data field mapping inconsistencies across the PRACA systems. ADAM is not addressing the system-wide architectural changes required of the Project systems.

Much of the data the Program requires and desires from the PRACA systems to make them more useful for Safety and Risk analysis are not consistent with the current Project/Element focus. Data currently gathered for the Program that is not necessary for local domain needs is generally handled with less care because its value and use are locally subjective. This is important to note for a number of reasons. It can lead to incorrect entries because the person making the entry does not understand the purpose of that data field in the PRACA form. Additionally, end users such as people doing trending and analysis may not understand the context in which the data were acquired and therefore why fields are being filled out in particular ways at operational sites. These mutual misunderstandings can produce incorrect data, which degrades the validity of any trending analyses using these data fields. The main commonality between the PRACA systems comes from the NSTS 08126 document. While each of the various PRACA systems comply with the current 08126 revisions, the Project-level PRACA systems have there own guiding requirements and documentation, of which the 08126 requirements play only a partial role. Data field naming conventions are solely left to the Project to implement in their local databases.

Streamlining efforts (efficiency, nurn-around time, work-flow) at the Project and domain level tend to resist addition of fields that impede the streamlining efforts. This is due in part because the local PRACA system is often used for a number of functions besides problem reporting and corrective action. Thus many individuals collecting the data may not see their job as connected to the SSP system-wide PRACA effort. This also contributes to opacity and misunderstandings of the roles and purpose of the SSP PRACA systems. 


\section{No PR.ACA System-wide Philosophy}

There is a lack of a clearly expressed vision for SSP PRACA and subsequent lack of a system-wide buy-in to the PRACA System philosophy. While the Space Shuttle Program expectation for PRACA is as a part of the PCASS, many view the PCASS as one of the several systems comprising PRACA data sources rather than as a PRACA end design goal. The problem was further complicated by a lack of clearly acknowledged PRACA owner For example, none could be identified or established during a visit to the SSP office in January 2000. Since January a PRACA owner has been identified, within the SSP as well as for the USA contract, by the JSC PET activity.

The creators or Project-level managers of the PRACA systems accept the SSP system-wide data resource goal but do not yet view PRACA data as a Program-wide resource that can be controlled and managed by the SSP office. An overall PRACA System as an organizational or technological entity does not exist. and was not required by the NSTS 08126 Revision G document. The JSC PET has rewritten and enhanced the NSTS 08126 document to a Revision $H$ and this is discussed later.

\section{Effect on PRACA Data}

The data collection workforce is not currently trained in PRACA system Program data needs and usage. The data collection complexity imposed on the local (domain) PRACA teams is not a primary consideration addressed in the PRACA requirements documentation. Because of the Project-focused development of the individual PRACA systems and their work practices, the resulting multiple definitions, levels and functions of PRACA lead to opacity between pars. That is, each user of a PRACA system understands it from the viewpoint of their Project/Element and their task. There is not a training process enabling a universally shared understanding about what PRACA data is, what its levels are, who has responsibility for which parts of PRACA data, and how to weigh the priorities of Program data collection against local rask schedules and deadlines.

We found that Project users, the engineers and technicians who originate reports, were more likely to understand PRACA data from the perspective of their center, or even of their specific job and its procedures, without understanding the larger implications for how the system(s) works and the SSP-wide service that it provides. This can lead to tensions berween the functions, and to the possibility of bad data being entered into the system.

For example, technicians at KSC who have found a nonconformance are required to fill out a PRACA Problem Report. Interim Problem Report or Discrepancy Report as a part of the process for dealing with nonconformances. However. the report s central use for them is to schedule the work of repair and assignment of safery constraints on other work, which may not be performed until the nonconformance is dispositioned. Many of the required data fields in the problem reporn form that are relevant for problem reporting are irrelevant to the scheduling process, and may be seen by users as demanding information to which they do not have ready access or which requires too much valuable time to answer.

One example of such a field is Vendor. It may be useful for developing trending data at the Program level, but this use is not clear to technicians. The field is not necessary for any of the work that the technicians do, nor is it particularly valuable to the engineers and schedulers who direct the technicians work. This confusion can lead to technicians filling in any value that they know will pass the inspection process, rather than artempting accuracy.

\section{Effect on Future Potential for PRACA}

The lack of a clearly articulated and adopted vision across the PRACA systems has an effect on the future potential for safety and risk analyses. Many of the visions expressed by NASA senior management for the ideal PRACA System include a prognostic capability with data search, navigation, and mining that extends across the Shutrle Program. Without some effort to promote unity amongst the systems or their data, the potential technical leverage from similar systems (e.g., the Aviation Safety Program) and NASA research Programs such as Design for Safety will be reduced. There are many opportunities for interaction and data sharing with the digitized Shutle components databases, commercial aviation maintenance planning and scheduling systems, and model-based reasoning systems that could significantly enhance the utility of the PRACA data for safety and risk analyses within the SSP. This is discussed further in the recommendation section of this report. 
NSTS 08126 Revision H Updates

The JSC PET has completed much of a rewrite and update to the NSTS 08126 document. This revision is appended as Revision $H$ to identify it as the successor to Revision $G$. Revision $H$ better reflects the desired scope and global functions expected of the SSP s PRACA system. The Space Shuttle Program Review Control Board is expected to approve 08126 Revision $\mathrm{H}$ in the summer of 2000.

Revision $\mathrm{H}$ now states that the goal of the PRACA system is to establish a process to continuously improve the safety and reliability of Space Shuttle hardware, software, and critical ground systems. The PRACA system will provide the SSP and all SSP Elements/Projects:

1) Accurate and immediate visibility into problems; and

2) An accurate historical database to support problem tsend analysis, provide failure history, support anomaly investigation. and to document corrective actions.

Revision $\mathrm{H}$ also recognizes that PRACA is only useful if the reported information is accurate and correct. It emphasizes that sufficient attention must be paid to insuring accuracy of the data comprising the problem report, failure summary, root cause analysis, and in/out-of-family screening.

NSTS 08126 Revision $H$ defines and enhances the SSP requirements for problem reporting, analysis, disposition, resolution, and trending. Problems that are documented in PRACA include: Space Shuttle hardware. Orbiter software discrepancies, main engine software discrepancies, Launch Processing System, Ground Support Equipment and Launch \& Landing facilities that support mission to mission processing of flight hardware. The Revision $\mathrm{H}$ document establishes:

1) Uniform criteria for reporting problems:

2) Requirements for problem disposition and closure:

3) Requirements for documentation of corrective action;

4) Requirements for problem documentation to support engineering and trend analysis;

5) Requirements to support logistics management; and

6) Definition of problem report data elements and terminology.

The NSTS 08126 Revision $H$ now addresses the requirements for extracting the data necessary for trend analysis and reporting. In addition, this latest version of the PRACA system requirements greatly improves and clarifies the requirements for the PRACA systems. Work is continuing on the PRACA data element definitions and establishing the database code translation tables to enable some mapping between the various PRACA systems data. These definitions and tables are to be included in the final version of NSTS 08126 Revision $H$.

\section{RECOMMENDATIONS}

In order to recommend modifications, upgrades, and enhancements to the SSP PRACA systems, we must establish two things: first, what PRACA currently is; and second, what PRACA should be. This study has endeavored to identify the current state of PRACA (i.e., what PRACA currently is). As for what PRACA should be, there are three fairly distinct mental pictures emerging from the team s interviews with the PRACA workforce, SSP management, and NASA senior management. These are:

1. The Project/Element domain expert view:

PRACA should remain a collection of relatively simple databases that support the work process and record-keeping functions. These databases are designed primarily to support the domain experts who are responsible for reporting Project/Element staus and trending to the SSP. The domain experts would prefer that the SSP continue to rely upon the domain experts for data extraction, filtering, analysis, interpretation and reporting from the PRACA databases and other sources.

2. The Fund Source (SSPNASAs Human Explomtion Entemprise) view:

PRACA is a multi-center data system that is vital to the SSP mission. The domain experts role in the PRACA system is consistent with the team problem resolution approach and is not seen as a porential problem. Ongoing reviews and relatively stable workforce will sustain the system s viability into the future. Additional work on PRACA should be justified based upon new capability. 
3. The NASA Information Management view:

PRACA should be a state-of-the-art data warehouse capable of data mining and advanced data analysis and trending using a simple and uniform point and click interface. The system should preclude data errors, incomplete problem tracking, and catch potential problems that might otherwise go undetected (e.g., escapes and diving catches). The system should reduce the sole dependence on domain experts and corporate knowledge, placing the power of top-ievel knowledge and information in the hands of anyone with access to the system via a simple user interface. Additionally, advanced data mining capabilities would support the SR\&QA analysts to improve the speed and accuracy of their assessments. With the Shutrle expected to fly another 25 years, the system architecture must be dynamic and capable of overcoming changes in workforce, technology, and flight rate. The system should be enhanced to provide a foundation enabling the future implementation of a safety and risk prognostics capability. The system should serve as a model and pathfinder for the Agency.

As we have noted previously, there was no overall SSP PRACA owner until recently and there still is no clear vision declaration for scope and functionality. The general vision of capability proposed in the NSTS 07700 volume XI is not being fulfilled with the present PRACA systems.

The Study team has chosen to present its recommendations with the assumption that the NASA Information Management view of the PRACA system should serve as a goal for the final system state and our recommendations. Sensitivity to the Project and Fund Source views was maintained but as secondary considerations. Given this attention and assumption guideline, this Study has identified several recommendations for modification, upgrade, and enhancement of the SSP PRACA System. The general recommendations are discussed as follows:

\section{Global Perspective}

The SSP-identified PRACA owner needs to make a global assessment of PRACA with both a short-term and long-term view. It is important to answer vision-defining questions such as:

- Is PRACA sufficient for the SSP needs? If so, for how long?

- Is PRACA to be a cutting edge information management system? Is it to serve as an example for Agency emulation?

- Is PRACA to look beyond SSP focus to leverage other safety and reporting systems? (Aviation Safety Program, Commercial aviation scheduling and planning systems, model-based reasoning systems, digitized Shuttle systems. other NASA PRACA systems, etc.)

- What is the evolving role for PRACA looking into the next 25 years?

- What is the relationship of PRACA to the changing NASA workforce? And how does that impact PRACA functionality over time?

- Is PRACA to be the foundation of a Safety and Risk Prognostics System for the SSP?

It is equally (if not more) important to answer design questions driving the requirements such as:

- Who are the customers for the system $s$ data?

- Who are the users of the system?

- Who are the managers of the system?

- What skill level(s) is expected of the owner, customers, users, and managers of the system?

- What is the security level of the data in the system, and what is the desired visibility in the community?

- How large a dependence on expert knowledge and human interpretation is acceptable?

- Is it permissible/desirable to use data outside of PRACA (and PCASS) or should PRACA be the sole source of data access?

- What are the roles of the Program office as an owner, user, and a customer of PRACA information?

- What is the role of PRACA at the data collection level? At the ProgramElement level?

Once these and other similar questions are answered, the SSP should clearly articulate its vision and train and/or inform personnel in all of the levels of the PRACA system. 
PRACA as an Element of Safety and Risk Prognustics

One of the unrealized possibilities for the PR.ACA database systems is as a foundation for a Safety and Risk Prognostics capability. Prognostics in this sense go beyond simple data trending. to provide a true predictive capability that could greatly enhance the decision-making capabilities of the SSP and the vafety of the Shuttle.

\section{Recommendation:}

- Establish a plan for PRACA system evolution that will enable the development of a future Safety and Risk Prognostics capability.

Impact:

- Improve the breadth and depth of the SR\&QA analyses performed by the experts in a given time frame, as well as ensure the high quality of the PRACA data for such analyses to be made.

- Provide a manager-level overview and quick look assessments of Shuttle safety and risk data.

- Enable SSP management to be more proactively involved and up-to-date on the performance and safety trade-offs for the Shuttle fleet.
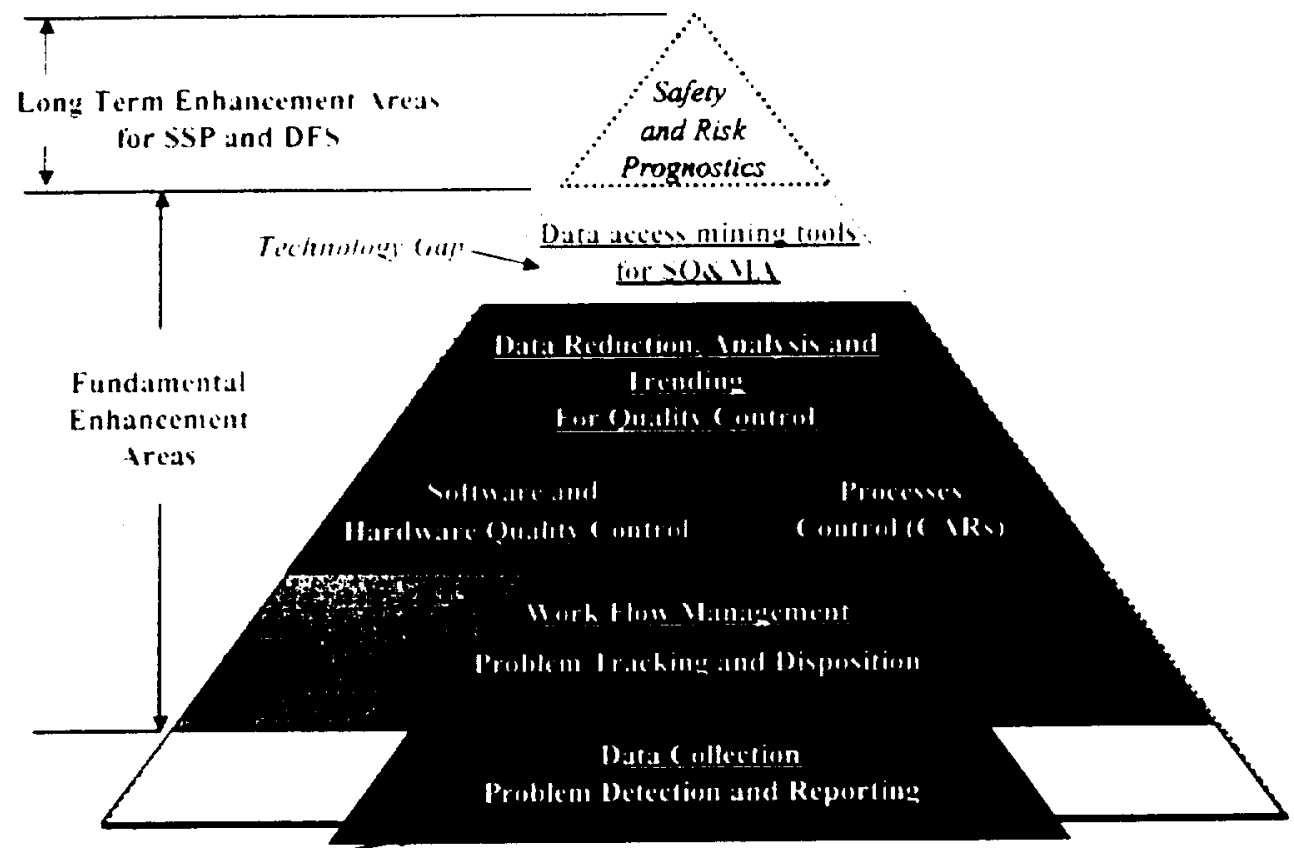

\section{Current PRACA Systems coverage}

Figure 1 - PRACA Data Use Concept

As noted in the figure above, a technology gap exists in the current PRACA technology pyramid. This technology gap is currently compensated for by the use of domain experts to manually search, interpret, filter, and process the data into knowledge for the SSP. This technology gap should be eliminated by:

1. Implementing improvements to the PRACA systems in the fundamental enhancements areas as shown in the figure

2. Implementing advanced data access, data mining. and unified user interfaces.

We believe that an improved and enhanced PRACA System could radically improve the breadth and depth of the SRQ\&MA analyses performed by the experts in a given time frame, as well as ensure the high quality of the PRACA data for such analyses to be made. Additionally, the future PRACA System would provide a manager-level overview and quick look 
assessments of Shuttle safety and risk data. This will enable SSP management to be more proactively involved and up-todate on the performance and safety trade-offs for the Shuttle fleet. Specific recommendations to do this are addressed in the Study s NASA Technical Memorandum.

\section{Update of PRACA Requirements}

Since the delivery of the SIAT repor in December 1999 and the initial ARC PRACA review in January 2000. the SSP created the PET to reassess and revise the NSTS 08126 requirements for PRACA. In conjunction with this activity. the USA Integrated PRACA Team has addressed many of the underlying processes and motivations for the various PRACA systems under its control. These activities do several important things:

- They unify some of the reporting requirements.

- They enhance some of the access requirements.

- They respond to multiple SSP and USA audits and reviews and address the SIAT report concerns and the informal ARC Srudy comments.

It is also critically important to answer the questions driving the requirements that have not been completely addressed by the aforementioned SSP and USA activities.

\section{PRACA Owners}

The creators or Project-level managers of the PRACA systems do not yet view PRACA data as a program-wide resource. An overall PRACA System as an organizational or technological entity does not exist, and was not required by the NSTS 08126 Revision G document. The JSC PET has rewritten and enhanced the NSTS 08126 document to a Revision H. Revision $\mathrm{H}$ better reflects desired scope and global functions required of the SSP S PRACA system. The Space Shuttle Program Review Control Board is expected to approve Revision $\mathrm{H}$ in the summer of 2000.

The SSP has identified a Shuttle Program PRACA Owner and USA has identified an internal PRACA owner. The team recommends that these owners take the action to declare the vision for the PRACA System and its evolution over the next 25 years. The vision declaration should create a concrete image in the minds of the PRACA workforce, from the data collectors through the SR\&QA analysts to the SSP management office.

Program-Level Access

As we discussed in a previous section of the study, the existing PRACA System is not sufficient for Program-level data mining and SQ\&MA assessment. The SSP currently meets the necessary constraint of having enough problem reporting data and insight by relying on a set of domain experts possessing extensive knowledge of the Shurle subsystems and the PRACA data, and who have access to additional non-PRACA (formal) data. These experts produce consolidated reports and summaries for the SSP office from which the SSP performs its tasks and formulates decisions. This sole dependence on expert knowledge and domain experts has shielded the SSP office from several PRACA deficiencies, including:

- PRACA data alone does not provide enough information for Program-level trending and data mining applications.

- There are multiple data sources on maintenance, repair, corrective actions and engineering dispositions (CARs, hazard reports, engineering databases, expert knowledge, etc.) not included in the PRACA systems (or even PCASS) and unavailable to the Program Office.

- Every PRACA system is unique and designed primarily for Project/Element (subsystem domain) use. Program use of the systems and their data are mainly handled as design patches to the systems.

- USA s ADAM is incomplete and unable to act as a data warehouse supporting cross database data mining. A proposed KSC/USA WebPCASS based upon the existing ADAM structure is currently proposed. This is a good first attempt to provide unified PRACA data and some associated information, but needs to go much farther.

- Generating SR\&QA reports is an extremely time and labor intensive activity requiring specialized knowledge and much massaging and cleaning of PRACA data.

To improve Program-level access to the PRACA data, we recommend that the current PRACA system be replaced or significantly upgraded. 


\section{Recommendations:}

- Clearly identify (list) the Progtam-level PRACA tasks from a Program-wide perspective

- Establish requirements for a PRACA System that performs SSP level PRACA tasks (data retrieval, mining and trending needs). This action should be performed without consideration of current PRACA capabilities.

- Design a PRACA System that satisfies these requirements.

- Either a) Implement this new system or b) Initiate a modernization activity to upgrade the current PRACA systems and designs to satisfy the requirements.

- Replace or enhance the existing WebPCASS proposal based upon the above decisions.

\section{PRACA Assessment Areas Recommendations}

As a foundation for the new PRACA system design, the team has identified specific deficiencies and recommended actions for each of the four assessment areas identified in our Study approach. It is important to note however, that we believe the Program-wide vision for PRACA (i.e., what PRACA should be ) must precede system technology changes.

With regard to the assessment area of functional capabilities and the upgrades recommended, it is our opinion that a PRACA Enhancement Project should

- Satisfy all the Program Offices task-based requirements (see previous section);

- Satisfy the ProjectElement (subsystem domain) work flow management requirements;

- Meet all NASA data security standards;

- Increase the user base through ease of access and intuitive user interface:

- Incorporate expert knowledge capture to assist in correct data interpretation and to reduce dependency on human corporate/institutional knowledge;

- Simplify system management and support requirements:

- Integrate with other Shuttle data sources to enable Data Mining in suppor of Risk Assessments:

- Provide advanced IT capabilities for Trending and Analysis in support of SRQ\&MA requirements;

Provide a migration path to a true safety and risk prognostics capability for the SSP.

\section{CONCLUSION}

The SSP PRACA System is an essential component to enable increased Shuttie safety and improved assessment of Shuttle readiness for flight. With the significant growth in the capabilities of Information Technology, the SSP PRACA System is poised to take advantage of the increased capabilities these advances provide. The SSP is motivated to increase the knowledge extraction capability of PRACA by using advanced IT tools for improved ease of access, greater breadth and depth of risk assessments, enhanced data quality and integrity, faster data mining and trending, and progression towards a true Safety and Risk Prognostic capability.

This Study has identified several areas where improvements in technology or implementation can enable a significant SSP PRACA improvement. In addition, the SSP PRACA System enhancement activity is capable of benefiting from other development activities such as Design for Safety (DfS) Program technology insertion, leverage from Aviation Safety Program developments, and other basic information technology enhancements coming from the Intelligent Systems Program.

We believe that an Agency-wide NASAIndustry team in conjunction with the SSP PRACA workforce can bring together the required expertise, knowledge base, and advanced IT capabilities necessary to achieve NASA s Information Management vision for PRACA. In so doing, PRACA will remain a critical and vital system, enabling a reduction in the risk and improvements in safety while supporting the Space Shuttle Program into the next decades.

\section{ACKNOWLEDGEMENTS}

The authors wish to thank all of the members of the PRACA Study team for all of their hard work. Ms. Linda Ham in the Space Shuttle Program office was particularly helpful and guided much of the areas of assessment. 


\section{REFERENCES}

1. Space Shuttle Program Review Control Board Action S06034IR5(3-1). Space Shuttle Program Office. NASA Johnson Space Center

2. Space Shuttle Problem Reporting and Corrective Action (PRACA) System Requirements, NSTS 08126. Revision G. Space Shurte Program Office. NASA Johnson Space Center

3. Shuttle Independent Assessment Team Report, NASA Ames Research Center, March 7, 2000.

4. The PRACA Pilot Study Assessment for the Space Shuttle Program . NASA Ames Research Center, Technical Memorandum in review, March 2001

5. NSTS Problem Reporting and Corrective Action (PRACA) to Program Compliance Assurance and Starus System (PCASS) Interface Definition Agreement, PCASS-IDA-0006, Space Shuttle Program Office. NASA Johnson Space Center

6. Space Shuttle Program Definition Requirements Volumes IV, V, XI, and XII, NSTS 07700. Space Shuttle Program Office. NASA Johnson Space Center

7. Advanced Data Acquisition \& Management (ADAM) Data Warehouse Project Plan, USA-96-dw-0001, United Space Alliance. 